\title{
Multiple evanescent white dot syndrome associated with retinal vasculitis
}

This article was published in the following Dove Press journal:

International Medical Case Reports Journal

21 September 2015

Number of times this article has been viewed

\section{Akihiro Takahashi \\ Wataru Saito \\ Yuki Hashimoto \\ Susumu Ishida}

Department of Ophthalmology, Hokkaido University Graduate School of Medicine, Sapporo, Japan
Correspondence: Wataru Saito Department of Ophthalmology, Hokkaido University Graduate School of Medicine, Nishi 7, Kita I5,

Kita-ku, Sapporo 060-8638, Japan

$\mathrm{Tel}+8$ I | | 7065944

Fax +8| || 7065948

Email wsaito@med.hokudai.ac.jp
Purpose: A recent study revealed thickening of the inner retinal layers in acute stage of multiple evanescent white dot syndrome (MEWDS); however, the pathogenesis is still unknown. We report two cases with MEWDS whose funduscopy showed obvious retinal vasculitis.

Methods: Case reports.

Results: Healthy myopic 16- and 27-year-old women were the cases under study. In both cases, funduscopic examination revealed multiple, faint, small, subretinal white dots at the posterior pole to the midperiphery and macular granularity oculus dexter. Retinal vascular sheathing was also observed at midperiphery. Late-phase fluorescein angiography revealed leakages corresponding to the vascular sheathing. Enhanced depth imaging optical coherence tomography revealed the discontinuity of the ellipsoid zone corresponding to the white dots and increased macular choroidal thickness. One month later, these white dots and retinal sheathing spontaneously resolved in both cases. Three months later, impairments of the outer retinal morphology and the visual acuity were restored.

Conclusion: These results suggest that retinal vasculitis possibly plays a role in the pathogenesis of thickened inner retinal layers in acute stage of MEWDS.

Keywords: enhanced depth imaging optical coherence tomography, choroidal thickness, inner retinal layer, retinal vascular sheathing

\section{Introduction}

Multiple evanescent white dot syndrome (MEWDS) is a unilateral chorioretinitis characterized by multiple small subretinal white dots extending from the posterior pole to the midperiphery, spontaneous resolution of the white dots within several weeks, and good visual prognosis, which has a propensity to affect young, myopic females. ${ }^{1,2}$ Spectral-domain optical coherence tomography (SD-OCT) revealed temporary impairments in outer retinal morphology that were restored together with improvements of visual function, ${ }^{3,4}$ indicating that outer retinal impairments mediate the visual function loss in patients with MEWDS. Moreover, our recent study with laser speckle flowgraphy revealed that choroidal blood flow velocity at the lesion area significantly increased with regression of MEWDS, and there was a correlation between the blood flow and visual functions, ${ }^{5}$ suggesting a close link between choroidal circulatory disturbance and the pathogenesis of MEWDS. However, there were a few reports regarding the inner retinal abnormalities on MEWDS.

Using SD-OCT C-scan, we demonstrated recently a subclinical increase of macular inner retinal layers together with a decrease of outer layers in the acute phase of MEWDS eyes. ${ }^{6}$ Similarly, Akiyama et al $^{7}$ reported that thickness of the ganglion cell 
layer and inner plexiform layer at the macula thinned with the regression of MEWDS. However, the pathogenesis of the inner layer thickening is still unknown, although complicated retinal phlebitis has been speculated as the reason. ${ }^{1,6,8} \mathrm{We}$ herein report two cases with MEWDS, whose funduscopy showed obvious retinal vasculitis.

Our institute's Review Board does not require approvals or patient consent for these case reports.

\section{Case reports}

\section{Case I}

A 27-year-old woman with mild myopia presented with blurred vision and photopsia of the right eye for 2 weeks. She had no particular medical or ocular histories.
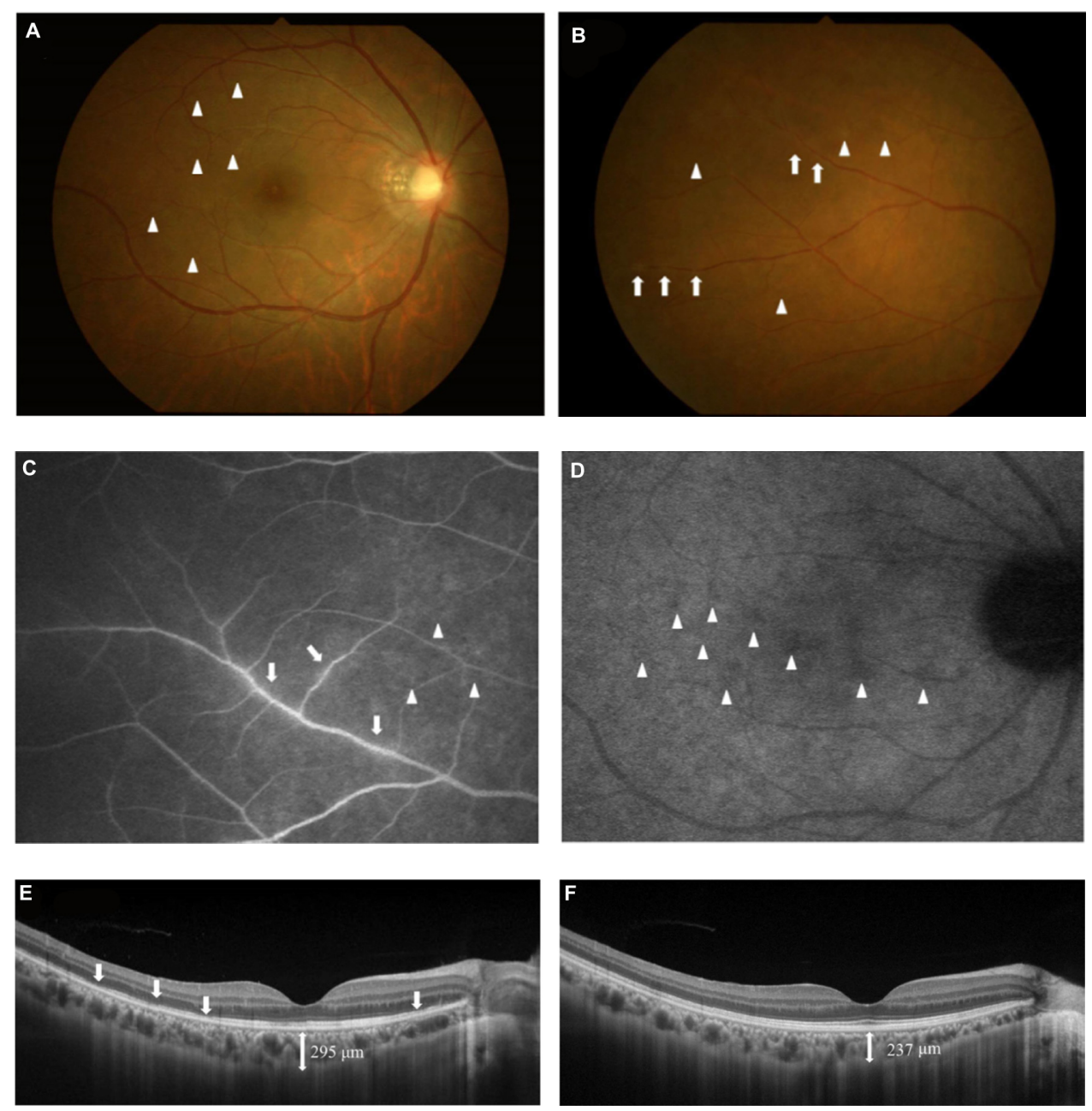

Figure I Photographs of the right eye in a patient with multiple evanescent white dot syndrome (Case I) at the initial visit (A-E) and 3 months later (F).

Notes: (A and B) Funduscopy showing multiple, faint, subretinal white dots extending from the posterior pole to the midperiphery (A and $\mathbf{B}$, white arrowheads), with foveal granularity. Retinal vascular sheathing was also observed in the temporal midperiphery (B, arrows). (C) Late-phase fluorescein angiography showing faint hyperfluorescence corresponding to the white dots (arrowheads) and retinal vascular wall staining with leakage corresponding to the retinal sheathing (arrows). (D) On late-phase indocyanine green angiography, multiple hypofluorescent spots scattered over a wider area than the white dots were seen (arrowheads). (E) A horizontal EDI-OCT image through the fovea revealed the discontinuity of ellipsoid zone at macular area corresponding to the white dots (arrows). SCT was $295 \mu \mathrm{m}$. (F) The ellipsoid zone at the macula spontaneously restored, and the SCT decreased to $237 \mu \mathrm{m}$.

Abbreviations: EDI-OCT, enhanced depth imaging optical coherence tomography; SCT, subfoveal choroidal thickness. 
the late-phase OD (Figure 1C, arrows). Indocyanine green angiography showed no abnormal appearance in the initial phase and multiple hypofluorescence at the wider area than the white dots (Figure 1D, arrowheads). Enhanced depth imaging optical coherence tomography (EDI-OCT) revealed the discontinuity of the ellipsoid zone at macular area OD (Figure 1E, arrows) and that the subfoveal choroidal thickness (SCT) was $295 \mu \mathrm{m}$. Humphrey perimetry (30-2 Swedish threshold interactive algorithm standard test) showed a mild blind spot enlargement $\mathrm{OU}$ (oculus uterque) (mean deviation [MD] value: $-1.83 \mathrm{~dB}$ OD and $-1.08 \mathrm{~dB}$ OS). Multifocal electroretinography (mfERG) showed entirely decreased amplitude, except normal central amplitude OD, and mildly decreased amplitude at the retinal site that corresponded to the peripapillary area OS. Infection screening, including tuberculosis, syphilis, human T-cell leukemia virus type 1 , and toxoplasmosis, showed negative results on QuantiFERON and serological testing or with antibodies for these agents. The chest X-ray was normal and serum angiotensin-converting enzyme was within normal limit. The patient received a diagnosis of MEWDS OD and was followed up with no treatment. One month after the first visit, the white dots and retinal vascular sheathing spontaneously resolved. After 3 months, the ellipsoid zone at the macula restored and the SCT decreased to $237 \mu \mathrm{m}$, together with increased BCVA of $1.5 \mathrm{OD}$ (Figure 1F). On Humphrey perimetry, the MD value improved to $-0.28 \mathrm{~dB}$ OD and $-0.69 \mathrm{~dB}$ OS.

\section{Case 2}

A 16-year-old woman with mild myopia complained of blurred vision and photopsia of the right eye for 1 month. The patient had no particular medical or ocular histories.

Her BCVA was 1.2 OU. Relative afferent pupillary defect was negative. Left eye was normal. Slit-lamp examination revealed no cells in the anterior chamber, but mild anterior vitreous cells OD. Funduscopic examination showed multiple, faint, small, subretinal white dots at the posterior pole to the midperiphery (Figure 2A and B, arrowheads), macular granularity, and retinal vascular sheathing at superotemporal midperiphery (Figure 2B, arrows). On FA, the hyperfluorescence was seen from the initial phase corresponding to the white dots (Figure 2C, arrowhead), with the staining of the optic disk and vascular leakages corresponding to the retinal vascular sheathing on the late phase (Figure 2C, arrow). On late-phase indocyanine green angiography, multiple spots of hypofluorescence were observed at the wider area despite no initial abnormal findings (Figure 2D, arrowheads).
EDI-OCT revealed segmental loss of the ellipsoid zone at areas of the white dots (Figure 2E, arrows), and the SCT was $341 \mu \mathrm{m}$. Goldmann perimetry showed a mild blind spot enlargement of $5 \times 10$ degree OD and no abnormality OS. On Humphrey perimetry, MD value was $-5.47 \mathrm{~dB}$ OD, and OS was unmeasured. In mfERG, entirely decreased amplitude except central normal amplitude was present OD, and the result was normal OS. Systemic screening, similar to what was done for Case 1 , showed negative results. She was diagnosed as having MEWDS OD and was followed up with no treatment. One month later, white dots and retinal vasculitis spontaneously disappeared. Three months after the initial visit, the ellipsoid zone at the macula restored and the SCT decreased to $319 \mu \mathrm{m}$ (Figure $2 \mathrm{~F}$ ). The BCVA remained unchanged. Twelve months after the initial visit, the MD value improved to $-0.24 \mathrm{~dB}$ OD. Thereafter, there were no recurrences of MEWDS during follow up.

\section{Discussion}

We encountered two MEWDS cases complicated with apparent retinal vasculitis at the midperipheral retina. To our knowledge, this is the first report of MEWDS cases which funduscopically showed obvious retinal vasculitis such as sarcoidosis.

Uveitis complicated with retinal vasculitis was considered as the differential diagnosis for our two cases. Sarcoidosis was eliminated based on the normal level of serum angiotensin-converting enzyme, normal chest X-ray, and the absence of anterior chamber inflammation. Uveitis associated with tuberculosis, syphilis, human T-cell leukemia virus type 1 , and toxoplasmosis were also differentiated from the negative results on serological test or antibodies for these agents. Based on our funduscopic findings, there might be a possibility that subretinal white dots existed along with retinal vessels accidentally. However, with FA, we detected the apparent evidence of retinal vasculitis corresponding to the retinal sheathing sites.

MEWDS eyes were reported to exhibit retinal periphlebitis, which was detected on FA in some cases, ${ }^{1,6,8}$ suggesting the involvement of inflammatory processes in the inner retina. These observations and our present results suggest that an increase of inner retinal layers in acute stage of MEWDS, as showed by our recent study, ${ }^{6}$ is due to accompanying retinal vasculitis. In our study examining inner retinal layer thickness of eight MEWDS eyes, indeed, only one case in which retinal phlebitis was detected on FA exhibited the maximal change in the inner retinal thickness during the course of the study, compared to those with no retinal vasculitis (data not shown). ${ }^{6}$ Two possibilities are considered as 

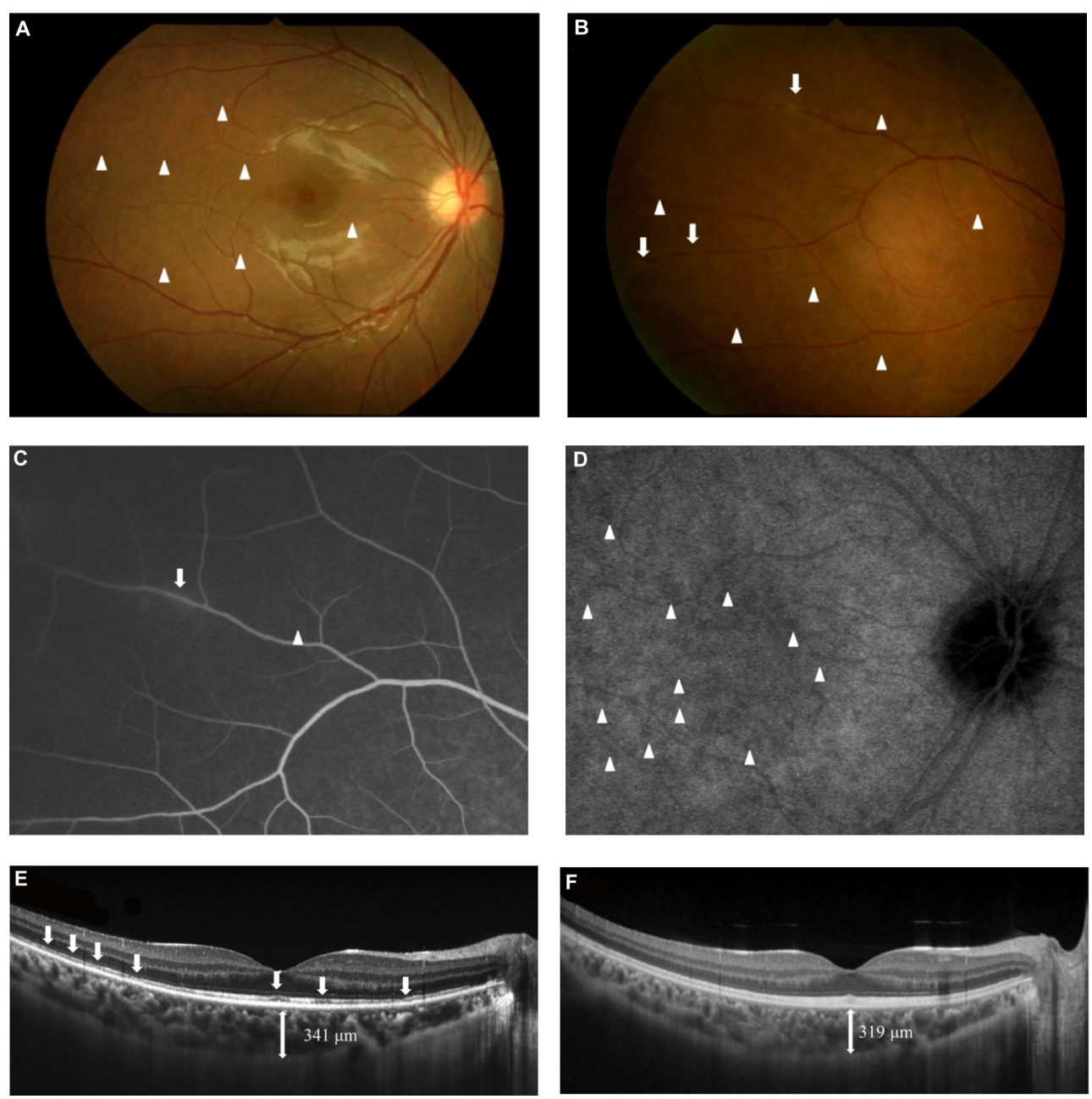

Figure 2 Photographs of the right eye in a patient with multiple evanescent white dot syndrome (Case 2) at the initial visit (A-E) and 3 months later (F). Notes: (A and B) Funduscopy showing multiple, faint, subretinal white dots extending from the posterior pole to the midperiphery (A and $\mathbf{B}$, white arrowheads), with foveal granularity. Retinal vascular sheathing was also observed in the temporal midperiphery (B, arrows). (C) Late-phase fluorescein angiography showing faint hyperfluorescence corresponding to the white dots (arrowhead) and vascular leakage from the retinal sheathing site (arrow). (D) Late-phase indocyanine green angiogram images showing multiple hypofluorescent spots scattered over a wider area than the white dots (arrowheads). (E) A horizontal EDI-OCT image through the fovea revealed the discontinuity of the ellipsoid zone at macular area corresponding to the white dots. SCT was $34 \mathrm{I} \mu \mathrm{m}$. (F) Macular ellipsoid zone spontaneously restored with the decrease of SCT $(319 \mu \mathrm{m})$.

Abbreviations: EDI-OCT, enhanced depth imaging optical coherence tomography; SCT, subfoveal choroidal thickness.

the cause of associated retinal vasculitis in MEWDS eyes; one is that inflammation extended from the outer layers to the inner layers, and the other is that vasculitis simultaneously occurs in inner layers apart from the inflammation in outer layers. Functionally, macular focal ERG revealed the significant reduction of not only a-wave but also b-wave and oscillatory potentials (OPs) in MEWDS eyes, and OPs were still reduced despite recovery of visual function and a- and b-waves during the course. ${ }^{9}$ Using mfERG, Cheng et $\mathrm{al}^{10}$ also observed reduced mfOPs in MEWDS eyes compared to the response from the fellow eyes, even in areas of the normal-appearing retina. Moreover, one patient showed attenuation of mfERG responses following mfOPs reduction. ${ }^{10}$ Morphologically, EDI-OCT showed abnormal hyperreflective lesions at ganglion cell layer in a MEWDS case with focal choroidal excavation. ${ }^{11}$ These observations suggest that the inner retinal layer changes in MEWDS occur independently with the outer layer abnormalities. Further studies are needed to investigate the relationship between MEWDS and retinal vasculitis.

In both of our cases, FA showed not only retinal vasculitis, but also the staining of the optic disk, suggesting that inflammation might extend to the optic disk as well. However, both cases involved negative relative afferent pupillary defect and decreased responses corresponding to the retinal sites with visual field defects on mfERG. These results and the abnormality of the ellipsoid zone that was observed at the acute stage suggest that the visual field impairment in these 
cases was caused by the outer retinal disorder but not by optic nerve involvement.

\section{Conclusion}

Two cases with MEWDS involved funduscopically obvious retinal vasculitis. These results may support the previous observation that showed an increase of inner retinal layer thickness in the acute stage of MEWDS, although further studies with a larger number of patients are needed to investigate the relationship between the inner retinal disturbance and retinal vasculitis in MEWDS.

\section{Disclosure}

The authors report no conflicts of interest in this work.

\section{References}

1. Jampol LM, Sieving PA, Pugh D, et al. Multiple evanescent white dot syndrome. Arch Ophthalmol. 1984;102(5):671-674.

2. Uy HS, Chan PS. Multiple evanescent white dot syndrome. In: Foster CS, Vitale AT, editors. Diagnosis and Treatment of Uveitis. Philadelphia, PA: Saunders Company; 2002:767-771.

3. Li D, Kishi S. Restored photoreceptor outer segment damage in multiple evanescent white dot syndrome. Ophthalmology. 2009;116(4): 762-770.
4. Hangai M, Fujimoto M, Yoshimura N. Features and function of multiple evanescent white dot syndrome. Arch Ophthalmol. 2009;127(10): 1307-1313.

5. Hashimoto Y, Saito W, Saito M, et al. Decreased choroidal blood flow velocity in the pathogenesis of multiple evanescent white dot syndrome. Graefes Arch Clin Exp Ophthalmol. Epub October 24, 2014.

6. Hashimoto Y, Saito W, Saito M, et al. Retinal outer layer thickness increases with regression of multiple evanescent white dot syndrome and visual improvement positively correlates with photoreceptor outer segment length. Acta Ophthalmol. 2014;92(7):591-592.

7. Akiyama $\mathrm{H}$, Itakura $\mathrm{H}, \mathrm{Li}$ D, et al. Retinal ganglion cell analysis in multiple evanescent white dot syndrome. BMC Ophthalmol. 2014;14:132.

8. Aaberg TM, Campo RV, Joffe L. Recurrences and bilaterality in the multiple evanescent white-dot syndrome. Am J Ophthalmol. 1985;100(1): 29-37.

9. Horiguchi M, Miyake Y, Nakamura M, et al. Focal electroretinogram and visual field defect in multiple evanescent white dot syndrome. $\mathrm{Br}$ J Ophthalmol. 1993;77(7):452-455.

10. Cheng JY, Luu CD, Yeo IY, et al. The outer and inner retinal function in patients with multiple evanescent white dot syndrome. Clin Exp Ophthalmol. 2009;37(5):478-484.

11. Hashimoto Y, Saito W, Noda K, Ishida S. Acquired focal choroidal excavation associated with multiple evanescent white dot syndrome observations at onset and a pathogenic hypothesis. BMC Ophthalmol. 2014; $14: 135$.
International Medical Case Reports Journal

\section{Publish your work in this journal}

The International Medical Case Reports Journal is an international, peer-reviewed open-access journal publishing original case reports from all medical specialties. Previously unpublished medical posters are also accepted relating to any area of clinical or preclinical science. Submissions should not normally exceed 2,000 words or

\section{Dovepress}

4 published pages including figures, diagrams and references. The manuscript management system is completely online and includes a very quick and fair peer-review system, which is all easy to use. Visit http://www.dovepress.com/testimonials.php to read real quotes from published authors.

Submit your manuscript here: http://www.dovepress.com/international-medical-case-reports-journal-journal 